\title{
CYTOKINE RESPONSE TO TYPICAL FIELD SPORTS PRACTICES IN ADOLESCENT ATHLETES
}

\author{
Alon Eliakim $^{1}$, Dan M. Cooper ${ }^{2}$, Dan Nemet ${ }^{1}$ \\ ${ }^{1}$ Child Health \& Sport Center, Pediatric Department, Meir Medical Center, \\ Sackler School of Medicine, Tel-Aviv University, Israel \\ ${ }^{2}$ Pediatric Exercise Research Center, University of California, Irvine
}

\begin{abstract}
The present study compares previous reports on the effect of "real-life" typical field individual (i.e. cross country running and wrestling - representing combat versus non-combat sports) and team sports (i.e. volleyball and water-polo - representing water and land team sports) training on pro (IL-6) and anti (IL-1 receptor antagonist - IL-1 ra) inflammatory mediators in male and female late pubertal athletes. An increase in IL-6 was found following each of the training sessions. In contrast, a significant increase in IL-1 ra was found only following the cross-country, wrestling and water-polo practices and not following the volleyball practices in both genders. There was no difference in the inflammatory response between individual and team sports practices. The inflammatory response to the typical practices was correlated with the practice-associated lactate change. The greatest increase in IL- 6 and IL-1ra occurred following contact sport practices, and was greater following land (wrestling) compared to the water (water-polo) practice suggesting that this increase may reflect muscle tissue damage and not necessarily training intensity. Further research is needed to better understand the influence of "real-life" typical training on exercise training adaptations of adolescent athletes.
\end{abstract}

Keywords: inflammatory mediators; adolescent athletes; sports training

\section{INTRODUCTION}

Exercise leads to simultaneous increase of antagonistic factors, and stimulates both anabolic components of the GH $\rightarrow$ IGF-I axis $[4,6]$, and catabolic proinflammatory cytokines $[7,10,11]$. Moreover, it was hypothesized that the 
fine balance between the anabolic and catabolic responses to exercise will determine the effectiveness of training. Anabolic response dominance will eventually lead to increased muscle mass and improved fitness, while prolonged dominance of the catabolic response, in particularly if combined with inadequate nutrition, may ultimately lead to overreaching and overtraining [3].

The majority of previous studies examined the effects of laboratory-based endurance-type exercise on the cytokine response. Interestingly, these studies suggested that the typical cytokine response to exercise is also antagonistic and includes an increase in pro-inflammatory mediators like IL-6 and IL-1 $\beta$ along with simultaneous increase of anti-inflammatory mediators like IL-1 receptor antagonist (IL-1ra) [7]. Surprisingly, very few studies examined the cytokine response to "real-life" exercise training in individual and team sports among adolescent athletes. This may be particularly important in adolescents, since repetitive catabolic and/or inflammatory response to exercise may be hazardous during puberty, a period characterized by rapid linear growth, muscle mass gain, and spontaneous anabolic hormones spurt. Therefore, the aim of the present study was to summarize and compare the effects of typical "real life" individual (i.e. cross country and wrestling) and team (i.e. volleyball and water-polo) sports training on IL-6 and IL-1 ra, two keypro- and anti-inflammatory mediators, in late pubertal male and female athletes. In addition, we determined training-associated changes in lactate levels, a commonly used marker of training intensity.

\section{MATERIALS AND METHODS}

We reviewed and reanalyzed our previous studies $[5,8,9,11]$ on the responses of hormonal and inflammatory markers to typical field practices in individual and team sports among late pubertal male and female athletes focusing on the inflammatory response. Characteristics of the participants and a brief description of the typical different practices are described below:

\section{Cross-country}

Eight elite female cross-country runners (mean age $16.75 \pm 0.5$ years, age range 15-18 years, Tanner stage for pubic hair 5) participated in the study. Participants were members of the university high school cross-country team and trained for 8-12 hours per week.

Training protocol: Warm-up (10 min) - a low intensity jog, followed by lower and upper body stretches. Continuous run (40-50 min) - the main workout consisted of 40 to 50 minutes of high-intensity running throughout 
the UCI Campus. The girls were motivated to continue running "all out" throughout the practice. Participants performed $10 \mathrm{~min}$ cool-down at the end of the practice.

\section{Wrestling}

Eleven elite male wrestlers (mean age $16.5 \pm 0.5$ years, age range $14-18.5$ years, Tanner stage for pubic hair 4-5) participated in the study. Participants were members of the University high-school wrestling team and trained for 8-12 hours per week.

Training protocol: Warm-up (15 min): Jogging, "stretch" exercise with sports-specific calisthenics such as push-ups and sit-ups. Technique Drills (15 min): The subjects performed typical wrestling skills including takedowns, escapes, pin combinations, and pin counters. The technique drills involved high-intensity exercise of short duration (6 to $10 \mathrm{sec}$ ). Situation wrestling $(15 \mathrm{~min})$ : The subjects were paired or placed in groups of 3 . Specific wrestling positions were assigned, and subjects wrestled from the given situation practicing a specific move and its counters. This involved exercise at maximal effort in bursts of 15 to $20 \mathrm{sec}$. "Iron Man" (15 min): Wrestlers were placed in groups of 5 or 6 with 1 wrestler in each group designated the "iron man". The "iron man" continuously wrestled facing a new partner every $30 \mathrm{sec}$. Designation of "iron man" rotated after about 3-4 min. Each wrestler was the "iron man" at least once during this drill. Live Wrestling (10 min): Each wrestler was paired with a partner of similar weight and ability. Each pair wrestled a full 6-minute match. Cool-down (10 min).

\section{Volleyball}

Twenty seven ( 14 males, mean age $16.3 \pm 1.1$ years, 13 females, mean age $16.0 \pm 0.4$ years) healthy elite, national team level Israeli junior volleyball players (age range 13.5-18 years, Tanner stage for pubic hair 4-5) participated in the study. All participants trained for 18-22 hours per week, played in the Israel premier junior volleyball league, belonged to the Israel national junior volleyball team and were members of the Israel National Academy for Gifted Athletes.

Training protocol: Each participant performed a typical one hour volleyball practice. Training consisted of 20 min dynamic warm-up which included jogging, stretching and running drills at sub-maximal speed (up to $80 \%$ of maximal speed), and additional $20 \mathrm{~min}$ of volleyball drills. The main part of the practice included seven repetitions of seven consecutive sprints from the back of the volleyball court to the net, maximal jump and 
a hit of the volleyball over the net in the end of each sprint. Each repetition lasted about 1.5 minutes with one minute rest to collect the balls between repetitions.

\section{Water-polo}

Ten elite female water polo players (mean age $15.1 \pm 0.3$ years, age range 14-16 years, Tanner stage for pubic hair 5) participated in the study. Participants were members of the University high-school water-polo team and trained for 8-12 hours per week.

Training protocol: Training consisted of 20 minutes dry-land and swimming warm-up. Twenty minutes of swimming conditioning including 10 minutes of high intensity drills. Fifteen minutes of leg drills (treading water, and leg swimming up and down the pool), 30 minutes of shooting (high intensity short swimming burst, vertical/horizontal water position change and shooting the ball to the goal), passing (between partners at increasing distances) and game drills (mimicking real game situations), and 5 minutes of cool-down.

\section{Blood Analysis}

Il-6 and IL-1ra levels in the different studies were analyzed using commercially available kits and lactate levels were determined using lactate analyzers. Blood samples were collected immediately before and at the end of each practice, and the effect of the typical practice on pro- and anti-inflammatory marker levels was calculated as the percent change from baseline levels.

\section{Statistical Analysis}

In each of the studies ANOVA was used to determine pre versus post exercise differences in lactate, IL-6 and IL-1 ra levels. Statistical significance was set at $\mathrm{p}<0.05$.

\section{RESULTS}

The effect of the different typical training sessions on IL-6, IL-1ra and lactate levels is summarized in Table 1 and Figures 1,2 and 3. Since the different training sessions were performed on different occasions, and cytokines were analyzed in different laboratories, the results are presented as percent change from baseline levels. However, for convenience of the readers, we also present the changes in absolute values. 
Table 1. Absolute changes in IL-6, IL-1ra and lactate levels following different types of typical ("real life") individual and team sports practices

\begin{tabular}{lcc|cc|cc}
\hline & \multicolumn{2}{c|}{$\begin{array}{c}\text { IL-6 } \\
\text { (pg/ml) }\end{array}$} & \multicolumn{2}{c|}{$\begin{array}{c}\text { IL-1 ra } \\
(\mathrm{pg} / \mathrm{ml})\end{array}$} & \multicolumn{2}{c}{$\begin{array}{c}\text { Lactate } \\
(\mathrm{mmol} / \mathrm{L})\end{array}$} \\
\cline { 2 - 8 } & PRE & POST & PRE & POST & PRE & POST \\
\hline Cross country Female & $2.8 \pm 1.1$ & $5.6 \pm 1.7^{*}$ & $366 \pm 105$ & $540 \pm 137^{*}$ & $1.4 \pm 0.2$ & $5.6 \pm 0.7^{*}$ \\
\hline Wrestling Male & $1.4 \pm 0.3$ & $10.9 \pm 1.4^{*}$ & $221 \pm 14$ & $425 \pm 40^{*}$ & $1.5 \pm 0.1$ & $9.0 \pm 1.3^{*}$ \\
\hline Volleyball Male & $1.2 \pm 0.6$ & $3.1 \pm 1.7^{*}$ & $296 \pm 86$ & $320 \pm 88$ & $3.1 \pm 0.2$ & $5.7 \pm 0.5^{*}$ \\
\hline Volleyball Female & $1.2 \pm 0.5$ & $2.5 \pm 1.1^{*}$ & $361 \pm 112$ & $381 \pm 146$ & $3.0 \pm 0.2$ & $5.1 \pm 0.3^{*}$ \\
\hline Water-polo Female & $1.9 \pm 0.5$ & $6.7 \pm 1.3^{*}$ & $290 \pm 58$ & $464 \pm 60^{*}$ & $0.9 \pm 0.1$ & $4.6 \pm 0.7^{*}$ \\
\hline
\end{tabular}

All training sessions were associated with a significant increase in lactate levels (Figure 1). Circulating IL-6 levels significantly increased following all of the training sessions (Figure 2). Circulating IL-1ra levels increased significantly following the cross-country, wrestling and water-polo practices, but not following the volleyball practices.

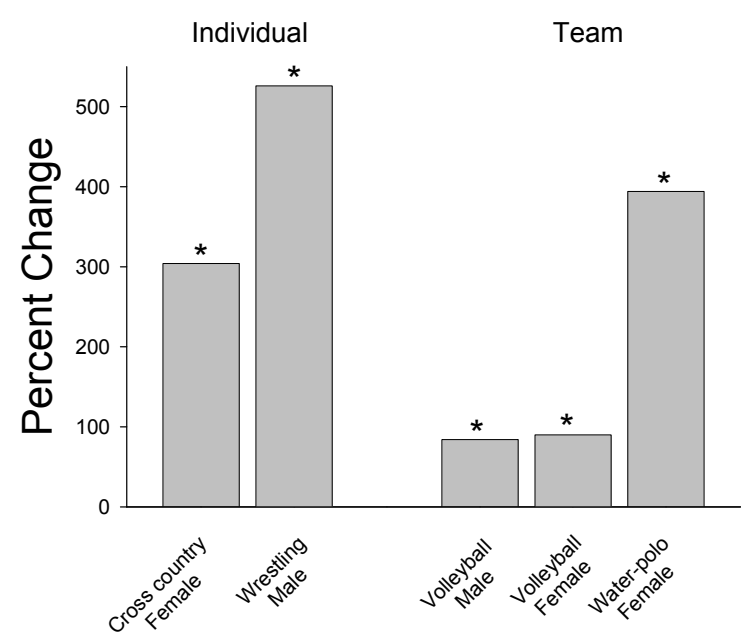

Figure 1. Percent changes in Lactate levels following different types of typical ("real life") individual and team sports practices 


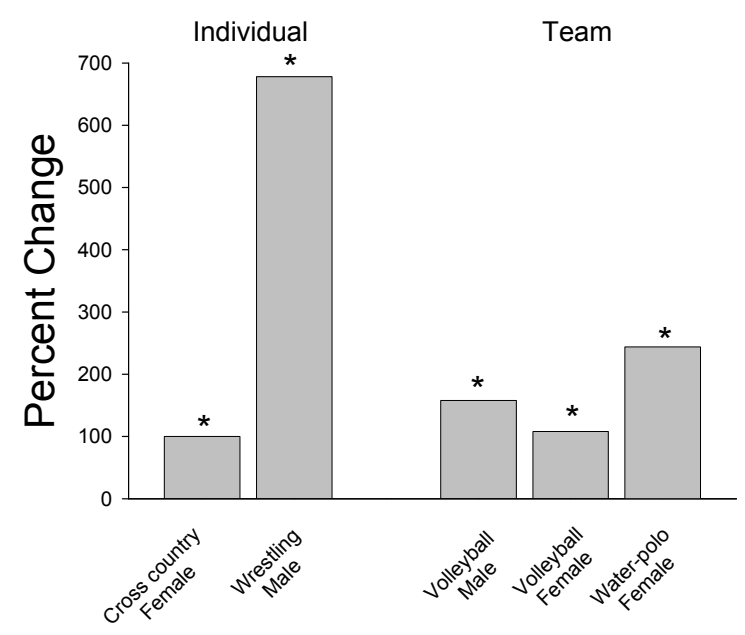

Figure 2. Percent changes in IL-6 levels following different types of typical ("real life") individual and team sports practices

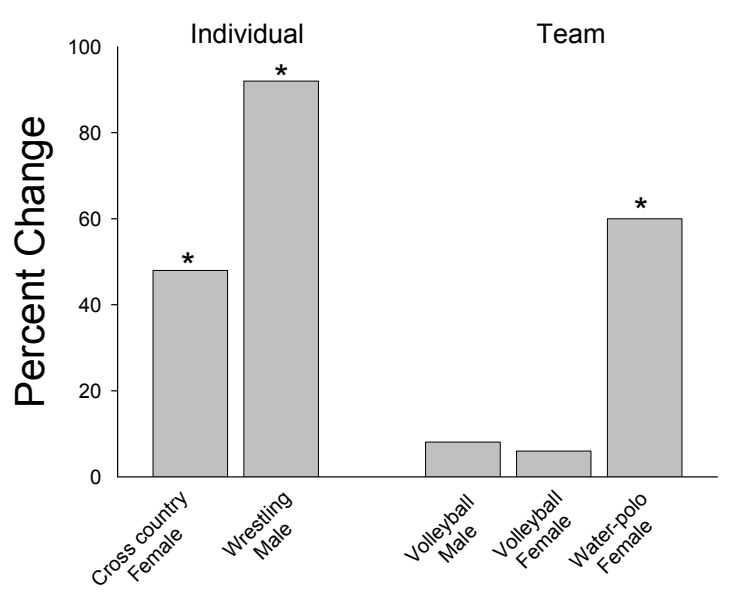

Figure 3. Percent changes in IL-1 ra levels following different types of typical ("real life") individual and team sports practices

\section{DISCUSSION}

The present study compared the effect of typical field individual and team sports training on pro- and anti-inflammatory markers in male and female late pubertal athletes. As previously described [1], we were interested in the effect of "real-life" typical field training sessions, therefore we were unable to control neither for the intensity of each practice nor for the fitness level of the participants in each session. In order to achieve some standardization, participants did not train during the day before the study, the duration of 
each practice was $60-80$ minutes, and the practice was performed during the initial phase of the training season when athletes are in relatively lower fitness level. All practice sessions were performed in the morning hours, and each typical practice included a warm-up, main part and a cool-down. A contact and non-contact sports were selected in the individual sport practices, and land and water sports were selected in the team sport practices. Training sessions were performed in different occasions and the inflammatory marker levels were analyzed by different laboratories using standardized kits. Despite these limitations, several interesting observations and conclusions can be drawn from this unique and rather infrequent comparison. We previously used the same approach to assess the effect of real life training sessions on GH and IGF, the main growth factors of the GH $\rightarrow$ IGF axis. The cross-country running practice and volleyball practice were associated with significant increases of circulating GH levels, while none of the practices led to a significant increase in IGF-I levels. We found that the magnitude (expressed also as percent change) of the GH response to the different practices was determined mainly by pre-exercise GH levels. No difference was found between individual and team sports practices in the trainingassociated GH response. Finally, the GH response to the different typical practices was not influenced by the practice-associated lactate change [1].

The major finding of the present study was that significant increase in IL-6 levels was found following each of the training sessions. In contrast, a significant increase in the anti-inflammatory mediator IL-1ra was found only following the cross-country, wrestling and water-polo practices and not following the volleyball practices in both genders. Therefore, our findings emphasize the notion that IL-6 is probably the most sensitive inflammatory cytokine to exercise [7]. The major source for the exercise-related IL-6 increase is the skeletal muscle [2]. IL-6 increases during exercise both with and without evidence of muscle damage. However, IL-6 is believed to play an important mediatory role in the inflammatory response needed for the exercise-associated muscle damage repair $[2,7]$ whether this trainingrelated muscle damage is prominent or only sub-clinical. This inflammatory response is coupled with an anti-inflammatory response represented in the present study by IL-1 ra. This emphasizes that the very complex exerciseassociated response of simultaneous antagonistic factors increase is not limited to anabolic and catabolic hormones, but also to inflammatory and anti-inflammatory cytokines.

Very few studies examined the effect of gender on the inflammatory response to exercise. Exercise-induced IL- 6 changes were similar between males and females following 90 minutes cycling at $65 \%$ of maximal aerobic 
power [13]. Interestingly, the exercise-induced change in IL-6 was approximately $80 \%$ greater in non-oral contraceptive users compared to oral contraceptive users during the follicular phase of the menstrual cycle, but was similar during the luteal phase of the menstrual cycle. Northoff et al. [12] examined the effect of $60 \mathrm{~min}$ run at $93 \%$ of the ventilatory anaerobic threshold on inflammatory gene expression in young adult men and women (during the follicular and luteal phases of the menstrual cycle). The authors found significant up-regulation of IL- 6 and down regulation of IL-1ra gene expression in women during the luteal phase of the menstrual cycle but not in women during the follicular phase or in men [12]. In our sample, the only practice that was studied in both males and females was the volleyball practice. We found a similar significant increase in IL-6 in both males and females, and that changes in IL-1ra were non-significant in both genders (Figures 2 and $3)$. Our female participants were non oral contraceptive users and were tested during the follicular phases of the menstrual cycle, and this may possibly explain the similar gender-related inflammatory marker responses in the present comparison. Further studies are needed to examine the effect of exercise on inflammatory markers in adolescent athletes during different stages of the menstrual cycle and between oral contraceptive users and non-users.

The present study included both individual and team sports typical practice sessions. It is often believed that individual sports' training is more demanding than the characteristically intermittent-type activity in team sports training. However, our results did not show differences in percent IL-6 change following the different typical individual and team sports practice sessions. This suggests that the IL- 6 response predominantly depends on the specific exercise task. Interestingly, however the only practices that were not associated with increases in the anti-inflammatory marker IL-1 ra were the volleyball practices in both males and female adolescents, although a significant IL-6 response was seen in both. Whether this indicates that a greater inflammatory response is needed following a typical volleyball practice, or that the anti-inflammatory response occurs later or in response to exercise associated inflammatory cytokines other than IL-6 (and therefore was not seen in the present study) needs to be further studied.

Previous studies that examined the effects of endurance-type exercise on hormonal and inflammatory mediators suggested that in order to stimulate secretion, the exercise input should be sufficient (e.g. above the lactic anaerobic threshold-LAT) to cause a substantial metabolic effect. In contrast to our previous report that changes in GH secretion were not related to changes in lactate levels following the similar different typical field practice sessions [1], changes in IL-6 and IL-1ra were correlated with changes 
in lactate levels. The greatest increases in lactate levels and inflammatory markers occurred following the wrestling and water-polo practices, and the lowest increases in lactate and inflammatory markers occurred following the volleyball practices in both males and females.

Finally, the greatest increases in IL-6 and IL-1ra occurred following contact sports practices, and were greater following the land (wrestling) practice compared to the water (water-polo) practice. This emphasizes once again the important mediatory role of these markers in the inflammatory response needed for exercise-associated muscle damage repair. However, the results may also suggest that the increase of these mediators following exercise reflect muscle damage and not necessarily training intensity, and therefore the interpretation of exercise-associated inflammatory markers increase should be done with caution. It is also possible that the reduced muscle forces generated in water medium can explain the lower exerciserelated muscle damage in contact water compared to land sports.

In summary, we compared our reports on the effect of "real-life" typical field individual (i.e. cross country and wrestling) and team (i.e. volleyball and water-polo) sports training on pro- and anti-inflammatory mediators in male and female late pubertal athletes. A significant increase in IL-6 was found following each of the training sessions. In contrast, a significant increase in the anti-inflammatory mediator IL-1ra was found only following the cross-country, wrestling and water-polo practices and not following the volleyball practices in both genders. There was no difference in the inflammatory response between individual and team sports practices. The inflammatory response to the typical practices was correlated with the practice-associated lactate change. The greatest increases in IL-6 and IL-1ra occurred following contact sport practices, and were greater following land (wrestling) compared to the water (water-polo) practice suggesting that this increase reflects muscle tissue damage and not necessarily training intensity. Further research is needed to better understand the effect of "real-life" typical training in exercise training adaptations of adolescent athletes.

\section{REFERENCES}

1. Eliakim A, Cooper DM, Nemet D. (2014) The GH-IGF-I response to typical field sports practices in adolescent athletes: a summary. Pediatr Exerc Sci, 26 (4): 428-433

2. Eliakim A, Nemet D. (2010) Exercise training, physical fitness and the growth hormone-insulin-like growth factor-1 axis and cytokine balance. Med Sport Sci, 55: $128-140$ 
3. Eliakim A, Nemet D. (2013) The endocrine response to exercise and training in young athletes. Pediatr Exer Sci, 25: 605-615

4. Eliakim A, Nemet D, Cooper DM. (2005) Exercise, training and the GH-->IGFI axis. In: Kraemer WJ, Rogol AD (eds). The Endocrine System in Sports and Exercise. Oxford, UK: Wiley-Blackwell, 165-179

5. Eliakim A, Portal S, Zadik Z, Rabinowitz J, Adler-Portal D, Cooper DM, Zaldivar, Nemet D. (2009) The effect of volleyball training on anabolic hormones and inflammatory markers in elite male and female adolescent players. J Strength Cond Res, 23: 1553-1559

6. Meckel Y, Eliakim A, Seraev M, Zaldivar F, Cooper DM, Sagiv M, Nemet D. (2009) The effect of a brief sprint interval exercise on growth factors and inflammatory mediators. J Strength Cond Res, 23 (1): 225-230

7. Nemet D, Eliakim A. (2010) Growth hormone-insulin-like growth factor-1 and inflammatory response to a single exercise bout in children and adolescents. Med Sport Sci, 55: 141-155

8. Nemet D, Eliakim A, Mills PJ, Meckel Y, Cooper DM. (2009) Immunological and growth factor response to cross-country training in adolescent females. J Pediatr Endocrinol Metab, 22: 995-1007

9. Nemet D, Mills PJ, Cooper DM. (2004) Effect of intense wrestling exercise on leucocytes and adhesion molecules in adolescent boys. Br J Sports Med, 38 (2): $154-158$

10. Nemet D, Oh Y, Kim HS, Hill M, Cooper DM. (2002) Effect of intense exercise on inflammatory cytokines and growth mediators in adolescent boys. Pediatrics, 110 (4): 681-689

11. Nemet D, Rose-Gottron CM, Mills PJ, Cooper DM. (2003) Effect of water polo practice on cytokines, growth mediators, and leukocytes in girls. Med Sci Sports Exerc, 35 (2): 356-363

12. Northoff H, Symons S, Zieker D, Schaible EV, Schäfer K, Thoma S, Löffler M, Abbasi A, Simon P, Niess AM, Fehrenbach E. (2008) Gender- and menstrual phase dependent regulation of inflammatory gene expression in response to aerobic exercise. Exerc Immunol Rev, 14: 86-103

13. Timmons BW, Hamadeh MJ, Devries MC, Tarnopolsky MA. (1985) Influence of gender, menstrual phase, and oral contraceptive use on immunological changes in response to prolonged cycling. J Appl Physiol, 99 (3): 979-985

\section{Corresponding author:}

Alon Eliakim MD

Pediatric Department

Meir Medical Center

59 Tchernichovski St.

Kfar-Saba, Israel

E-mail: eliakim@zahav.net.il

Tel: +972-9-7471596, fax: +972-9-7471303 